\title{
Evaluating the pathogenic potential of genes with de novo variants in epileptic encephalopathies
}

\author{
Na He, MD ${ }^{1}$, Zhi-Jian Lin, MD ${ }^{1}$, Jie Wang, MD ${ }^{1}$, Feng Wei, MD ${ }^{1}$, Heng Meng, MD², \\ Xiao-Rong Liu, MD ${ }^{1}$, Qian Chen, MD, PhD ${ }^{3}$, Tao Su, PhD ${ }^{1}$, Yi-Wu Shi, PhD ${ }^{1}$, Yong-Hong Yi, MD ${ }^{1}$ and \\ Wei-Ping Liao, MD, $\mathrm{PhD}^{1}$
}

Epileptic encephalopathies comprise a group of catastrophic epilepsies with heterogeneous genetic etiology. Although nextgeneration sequencing techniques can reveal a number of de novo variants in epileptic encephalopathies, evaluating the pathogenicity of these variants can be challenging. Determining the pathogenic potential of genes in epileptic encephalopathies is critical before evaluating the pathogenicity of variants identified in an individual. We reviewed de novo variants in epileptic encephalopathies, including their genotypes and functional consequences. We then evaluated the pathogenic potential of genes, with the following additional considerations: (1) recurrence of variants in unrelated cases, (2) information of previously defined phenotypes, and (3) data from genetic experimental studies. Genes related to epileptic encephalopathy revealed pathogenicity with distinct functional alterations, i.e., either a gain of function or loss of function in the majority; however, several genes warranted further study to confirm their pathogenic potential. Whether a gene was associated with distinct phenotype, the genotype (or functional alteration)--phenotype correlation, and quantitative correlation between genetic impairment and phenotype severity were suggested to be specific evidence in determining the pathogenic role of genes. Data from epileptic encephalopathy-related genes would be helpful in outlining guidelines for evaluating the pathogenic potential of genes in other genetic disorders.

Genetics in Medicine (2019) 21:17-27; https://doi.org/10.1038/s41436018-0011-y

Keywords: de novo variant; epilepsy; epileptic encephalopathy; next generation sequencing; pathogenicity

\section{INTRODUCTION}

Epileptic encephalopathies embody a group of age-dependent devastating syndromes, which can be characterized by refractory seizures, severe electroencephalography (EEG) abnormalities, and psychomotor development delay or decline. Approximately $40 \%$ of new-onset epilepsies before the age of 3 years can be attributed to epileptic encephalopathy, ${ }^{1}$ for example, Ohtahara syndrome, West syndrome, and Dravet syndrome. Recent studies have demonstrated the important role of genetic determinants underlying epileptic encephalopathy. ${ }^{2}$ Most epileptic encephalopathy cases are clinically sporadic, coinciding with frequently identified de novo variants. ${ }^{2}$ Although a number of de novo genetic variants have been detected in patients with epileptic encephalopathy with the application of next-generation sequencing (NGS), the causality of these variants remains elusive. Recently, several standards and guidelines have been recommended for the interpretation of sequence variants. ${ }^{3,4}$ Generally, the pathogenicity of sequence variants should be evaluated at the variant level, as well as at the gene level. ${ }^{3}$ At the gene level, the association between a gene and the disease, i.e., the pathogenic potential of the gene, which refers to the disease-causing potential if a variant occurred in this gene, should be evaluated. Further, implications, such as the potential pathogenic genotype, functional alteration, and genic subregion effect ${ }^{5}$ should also be assessed. Generally, assessment of gene-level implications is required before evaluating the pathogenicity of variants identified in an individual. ${ }^{4}$ In most situations, however, it is hard to determine the pathogenic potential of a gene based on data from an individual study, especially for genes with de novo variants that lack familial information of cosegregation. In the present study, we reviewed de novo variants identified in patients with epileptic encephalopathy and evaluated the pathogenic potential of the genes, with the aim of providing a basis for assessment of the pathogenicity of variants in a clinical or genetic consulting practice. We further assessed the evidence for evaluating the pathogenic potential of epileptic encephalopathy-related genes, with an expectation of enabling the formulation of guidelines for evaluating the pathogenic potential of genes.

\footnotetext{
${ }^{1}$ Institute of Neuroscience and Department of Neurology of the Second Affiliated Hospital of Guangzhou Medical University, Key Laboratory of Neurogenetics and Channelopathies of Guangdong Province and the Ministry of Education of China, Guangzhou, China; ${ }^{2}$ Department of Neurology, the First Affiliated Hospital, Jinan University, Clinical Neuroscience Institute, Jinan University, 613 Huangpu Avenue West, Guangzhou, Guangdong Province 510630, China; ${ }^{3}$ McGovern Institute for Brain

Research, Department of Brain and Cognitive Sciences, Massachusetts Institute of Technology, Cambridge, MA 02139, USA. Correspondence: W.-P. Liao (wpliao@163.net, wpliao@gzhmu.edu.cn)

These authors contributed equally: $\mathrm{Na} \mathrm{He}$, Zhi-Jian Lin.
}

Submitted 17 October 2017; accepted: 20 March 2018

Published online: 12 June 2018 


\section{STRATEGY OF LITERATURE SEARCH AND ANALYSIS}

Relevant articles were identified with searches on PubMed until November 2017. According to the classification defined by the International League Against Epilepsy (1989, 2001, and 2010), the following epileptic encephalopathy syndromes were taken into account: early myoclonic encephalopathy, Ohtahara syndrome, West syndrome (or infantile spasms), Dravet syndrome (or severe myoclonic epilepsy in infancy), Lennox-Gastaut syndrome, Landau-Kleffner syndrome, epilepsy with myoclonic-atonic seizures (EMAS), epileptic encephalopathy with continuous spike-and-wave during sleep (ECSWS), and epilepsy of infancy with migrating focal seizures (EIMFS). Regarding the technique, studies that employed any of the three major NGS technologies, i.e., genome sequencing, exome sequencing, and targeted sequencing (restricted in some interesting candidate genes), were included. We searched literature on the PubMed database using two keywords, namely, a combination of each syndrome and NGS technological aspect, e.g., "epileptic encephalopathy" and "next-generation sequencing." Using the same strategy, we searched the Ovid database and cross-checked the findings with the results from PubMed. We also searched OMIM (https://www.ncbi.nlm.nih.gov/omim/) for epileptic encephalopathy-associated genes. ClinVar (https://www. ncbi.nlm.nih.gov/clinvar/) and HGMD (http://www.hgmd. cf.ac.uk/ac/index.php) were consulted for any missed variants. To observe the frequencies of variant in different genes, studies on identification of variants in a given gene by conventional sequencing method were excluded (Supplementary Figure S1 online).

We analyzed the genotype, functional alteration (funotype the type of functional consequences of a variant), ${ }^{6}$ and phenotype (subtype of epileptic encephalopathy) of de novo gene variants identified in epileptic encephalopathy. Missense variants were further specified by functional alterations that were classified as gain of function (GOF), loss of function (LOF), which referred to the complete loss of function, or partial loss of function, which was used to denote mutants with residual activities, as outlined in our previous reports. ${ }^{6,7}$ Conversely, the functional consequence of variants that caused gross protein malformations and mostly led to haploinsufficiency, including truncating variants (nonsense and frameshifting), splice-site variants, and those causing genomic rearrangement, was referred to as destructive variants. $^{7}$

The pathogenic potential of each gene was evaluated with the following considerations: (1) recurrence of variants in unrelated cases; (2) information of previously defined phenotypes of the genes, including inheritance pattern, and the correlation between genetic impairment and clinic severity; and (3) data from genetic experimental studies. Evidence for evaluating the pathogenic potential of epileptic encephalopathy-related genes was analyzed, from which a guideline was outlined.

\section{PATHOGENIC POTENTIAL OF THE GENES An overview}

In total, 151 initial publications identifying de novo gene variants in epileptic encephalopathy were retrieved, in which 134 genes and 704 de novo variants were reported.

Regarding replication, 644 de novo variants were identified in 74 genes, which were suggested to be associated with epileptic encephalopathy by variants identified in two or more unrelated cases in each gene (Supplementary Table S1 online). These included 37 genes that were validated by independent studies, as well as recurrent variants in unrelated cases within a cohort; the remaining genes were validated either by single cases in more than one independent study or recurrent cases in a single study. All these genes are expressed in the brain. Fifty-two of these genes were previously reported to be associated with brain dysfunction phenotypes, among which epilepsies were the most frequent (48/52). Details of the variants and their original references are summarized in Supplementary Tables S2-6 online. These genes were subjected to further evaluation of pathogenic potential in epileptic encephalopathy (Supplementary Figure S1 online).

For the remaining 60 genes, only one de novo variant was identified in a single case for each gene (Supplementary Table S7 online). Their plausible relevance to epileptic encephalopathy was generally based on previous data showing a possible association with epilepsy in the original studies. These genes are either epilepsy-causing, or epilepsy-related, or potentially epilepsy-associated. ${ }^{8}$ Considering that each individual carries at least one de novo variant in the exome or approximately 100 de novo variants in the whole genome, ${ }^{9}$ the pathogenic potential of these genes in epileptic encephalopathy was not evaluated further.

We summarized the variants and their genotype, functional alteration, and sub-phenotype of epileptic encephalopathy, referred to the previously defined phenotypes of these genes (Tables 1,2, Supplementary Tables S8, S9 online), and evaluated the association between each gene and the epileptic encephalopathy.

\section{Sodium channel genes}

$S C N 1 A, S C N 2 A$, and SCN8A, which encode the principal a subunits of sodium channels $\mathrm{Na}_{\mathrm{v}} 1.1, \mathrm{Na}_{\mathrm{v}} 1.2$, and $\mathrm{Na}_{\mathrm{v}} 1.6$, respectively, have been suggested to be associated with epileptic encephalopathy, as evidenced by multiple sources (Table 1, Supplementary Table S2 online).

Variants in the SCN1A gene were initially identified in mild familial generalized epilepsy with febrile seizures plus, ${ }^{10}$ and were later confirmed to be the major cause of severe Dravet syndrome, ${ }^{6}$ a typical form of epileptic encephalopathy. Previous studies have demonstrated a close correlation between genotype (or funotype) and phenotype of SCN1A variants. ${ }^{6}$ Using NGS technologies, 78 SCN1A de novo variants have been identified in 87 patients with epileptic encephalopathy (Table 1). Existing data confirmed that these variants were destructive or caused LOF. SCN1A de novo variants were also identified in $7.0 \%(8 / 115)$ of patients with 
Table 1 Genotype, functional alteration, and phenotype of variants in ion channel genes in epileptic encephalopathies

Gene

Genotype (variant no.) Funotype (variant EE phenotype (case no.) no.)

\begin{tabular}{|c|c|c|c|c|}
\hline \multicolumn{5}{|c|}{ Sodium channel } \\
\hline \multirow[t]{4}{*}{ SCN1A } & Missense (42) & GOF $(0)$ & / & GEFS + (AD mostly) \\
\hline & & $\operatorname{pLOF}(2), \operatorname{LOF}(2)$ & DS (6) & PEFS + (AD or de novo), DS (de novo mostly) \\
\hline & & & DS (18), EIMFS (5), LGS (4), uEE (14) & \\
\hline & $\begin{array}{l}\text { Truncating (26), splice } \\
\text { (10) }\end{array}$ & Destructive (36) & DS (28), LGS (4), EMAS (1), uEE (7) & \\
\hline \multirow[t]{4}{*}{ SCN2A } & Missense (53) & GOF (2) & OS (2), uEE (3) & BFNS (AD), uEE (de novo) \\
\hline & & $\operatorname{pLOF}(0)$ & & \\
\hline & & UD (51) & OS (12), WS (9), EIMFS (1), LGS (1), UEE & \\
\hline & Truncating (2) & Destructive (2) & LGS (1), UEE (1) & \\
\hline \multirow[t]{4}{*}{ SCNBA } & Missense (45) & GOF (8) & $\mathrm{DS}(1), \mathrm{UEE}(21)$ & I \\
\hline & & $\operatorname{pLOF}(1), \operatorname{LOF}(1)$ & UEE (2) & I \\
\hline & & UD (35) & $\operatorname{DS}(2), \operatorname{EIMFS}(1), \operatorname{LGS}(1), \operatorname{WS}(1), \operatorname{uEE}(36)$ & BFIS (AD) \\
\hline & Truncating (1), splice (1) & Destructive (2) & uEE (2) & I \\
\hline \multicolumn{5}{|c|}{ Potassium channel } \\
\hline KCNA1 & Missense (2) & UD (2) & UEE (2) & EA/myokymia ${ }^{a}(A D)$ \\
\hline \multirow[t]{4}{*}{ KCNA2 } & Missense (12) & GOF (3) & UEE (8) & GE (de novo) \\
\hline & & G-LOF (3) & uEE (5) & \\
\hline & & LOF (4) & DS (2), EMAS (1), uEE (4) & FE (de novo), $H_{S P}{ }^{a}(A D)$ \\
\hline & & UD (2) & UEE (2) & I \\
\hline \multirow[t]{3}{*}{ KCNB1 } & Missense (9) & LOF (6) & WS (1), uEE (7) & I \\
\hline & & UD (3) & uEE (4) & I \\
\hline & Truncating (1) & Destructive (1) & WS (1) & / \\
\hline \multirow[t]{4}{*}{ KCNQ2 } & Missense (64) & GOF (4) & WS (5), EME (1), UEE (3) & I \\
\hline & & $\operatorname{pLOF}(4)$, LOF (3) & OS (2), WS (2), UEE (9) & BFNS/uEE (AD mostly) \\
\hline & & UD (53) & OS (14), WS (7), uEE (41) & BFNS (AD mostly) \\
\hline & Truncating (2) & Destructive (2) & WS (1), UEE (1) & BFNS (AD mostly) \\
\hline \multirow[t]{2}{*}{ KCNT1 } & Missense (29) & GOF (10) & $\operatorname{EIMFS}(24)$, WS (2), uEE (3) & NFLE (AD mostly) \\
\hline & & UD (19) & EIMFS (13), uEE (6) & / \\
\hline \multicolumn{5}{|c|}{ Calcium channel } \\
\hline CACNA1A & Missense (5) & UD (5) & EIMFS (1), LGS (1), uEE (5) & $\mathrm{EA}^{\mathrm{a}}(\mathrm{AD}), \mathrm{FHM}^{\mathrm{a}}(\mathrm{AD})$ \\
\hline \multicolumn{5}{|c|}{ NMDA receptor } \\
\hline \multirow[t]{2}{*}{ GRIN1 } & Missense (7) & $\operatorname{pLOF}(1), \operatorname{LOF}(2)$ & WS (1), uEE (2) & Severe $I^{\mathrm{a}}$ (de novo) \\
\hline & & UD (4) & UEE (5) & Severe ID (de novo) \\
\hline \multirow[t]{4}{*}{ GRIN2A } & Missense (3) & GOF (1) & uEE (1) & FE (AD) \\
\hline & & LOF (1) & $\operatorname{LKS}(1)$, uEE (1) & FE (AD or de novo) \\
\hline & & UD (1) & UEE (2) & FE (AD or de novo) \\
\hline & Truncating (0) & Destructive (0) & 1 & FE (AD mostly) \\
\hline \multirow[t]{3}{*}{ GRIN2B } & Missense (12) & GOF (2) & WS (2) & FE (de novo) \\
\hline & & UD (10) & WS (7), LGS (1), UEE (2) & I \\
\hline & Splice (1) & Destructive (1) & uEE (1) & I \\
\hline GRIN2D & Missense (1) & GOF (1) & UEE (2) & I \\
\hline \multicolumn{5}{|c|}{ Cyclic nucleotide-gated ion channel } \\
\hline \multirow[t]{3}{*}{ HCN1 } & Missense (7) & GOF (3) & UEE (3) & I \\
\hline & & LOF (2) & UEE (2) & / \\
\hline & & UD (2) & UEE (2) & IGE (unknown) \\
\hline
\end{tabular}

Variants in GABA-A receptor genes are summarized in Supplementary Table S 8 online, and all known functional alterations in these genes were LOF. Genes with pathogenic potential to be confirmed are not listed here. Details in Supplementary Table S2-4 online

$A D$ autosomal dominant, BFIS benign familial infantile seizures, BFNS benign familial neonatal seizures, DS Dravet syndrome, EA episodic ataxia, EE epileptic encephalopathy, EIMFS epilepsy of infancy with migrating focal seizures, EMAS epilepsy with myoclonic-atonic seizures, EME early myoclonic encephalopathy, FE focal epilepsy, FHM familial hemiplegic migraine, GE generalized epilepsy, GEFS+ generalized epilepsy with febrile seizures plus, G-LOF gain- and loss-of-function, GOF gain of function, HSP hereditary spastic paraplegia, ID intellectual disability, IGE idiopathic generalized epilepsies, LGS Lennox-Gastaut syndrome, LKS Landau-Kleffner syndrome, LOF loss of function, NFLE nocturnal frontal lobe epilepsy, OS Ohtahara syndrome, PEFS+ partial epilepsy with febrile seizures plus, pLOF partial loss of function, UD functional alteration not determined missense, UEE unspecified epileptic encephalopathy, WS West syndrome

aPhenotype with seizures

Lennox-Gastaut syndrome and occasionally in other epileptic encephalopathies. These findings confirmed that LOF of $\mathrm{Na}_{\mathrm{v}} 1.1$ is the main cause of Dravet syndrome and highlighted its possible role in other forms of epileptic encephalopathy.

SCN2A variants were first identified in families with benign familial neonatal-infantile seizures. ${ }^{11}$ Recent large-scale sequencing initiatives have identified 55 de novo variants in 69 sporadic cases with epileptic encephalopathy (Table 1), including several hotspot variants. Most $S C N 2 A$ variants were missense. However, both LOF and GOF were detected in mutants from either benign familial neonatal-infantile seizures or epileptic encephalopathy, with no distinct difference in functional alternation between them. ${ }^{12}$ In the mouse model, heterozygotes for the Scn $2 a$ knockout did not show seizures. ${ }^{13}$ Therefore, the functional alterations caused by heterozygous $S C N 2 A$ variants, especially LOF variants, cannot explain their pathogenicity in epileptic encephalopathy.

SCN8A was a novel gene that was reported to be associated with epileptic encephalopathy, with the identification of 47 de novo variants (Table 1), which were all missense variants except two. The R850, R1617, A1650, and R1872 residues were four hot spots, with variants at these residues accounting for $34.3 \%$ (23/67) of patients with epileptic encephalopathy (Supplementary Table S2 online). Electrophysiological 
alterations were determined in 10 mutants, of which 8 showed a GOF effect, including R1617 and R1872. These findings suggest that SCN8A variants with GOF are potentially pathogenic. Two mutants displayed LOF or partial LOF effect. However, Scn8a-null heterozygotes mice were seizure resistant. ${ }^{14}$ Therefore, the role of LOF of SCN8A in the pathogenesis of epileptic encephalopathy remains to be confirmed.

\section{Potassium channel genes}

Potassium channels compose a large and diverse superfamily of ion channels. Voltage-gated potassium channels $\left(\mathrm{K}_{\mathrm{v}}\right)$ regulate outward $\mathrm{K}^{+}$currents that contribute to membrane repolarization and hyperpolarization, thus limiting neuronal excitability. ${ }^{15}$ Genes KCNA1, KCNA2, KCNB1, KCNQ2, and KCNT1 have been reported to be associated with epileptic encephalopathy (Table 1, Supplementary Table S3 online).

Twelve missense variants in KCNA2 were identified in 22 patients with epileptic encephalopathy (Table 1). R297, T374, and $\mathrm{P} 405$ residues appeared to be hotspots for variants. Functional studies on the Q213X, I263T, G398C, and P405L variants demonstrated a dramatic reduction of current amplitudes (complete LOF) and a dominant-negative effect on wildtype $\mathrm{K}_{\mathrm{v}} 1.2$ channels. In contrast, the E157K, R297Q, and $\mathrm{L} 298 \mathrm{~F}$ variants induced a drastic GOF that caused permanent channel opening. Most patients with LOF variants had focal seizures that responded well to antiepileptic drugs and half of them became seizure-free. ${ }^{16}$ Patients with GOF variants, however, presented generalized seizures and had less favorable outcomes with more severe intellectual disability, ${ }^{16}$ suggesting a funotype-phenotype correlation. However, the detailed mechanism for GOF of the $\mathrm{K}_{\mathrm{v}} 1.2$ channel that leads to epilepsy remains unclear.

Nine KCNB1 missense and one truncating variants were reported in unrelated patients with epileptic encephalopathy (Table 1). Functional studies were performed in six variants and revealed a LOF effect, ${ }^{17,18}$ explaining their roles in epileptic encephalopathy.

More than 80 variants in KCNQ2 have been previously reported, mainly in patients with benign familial neonatal seizures. ${ }^{19}$ Additionally, 66 de novo missense variants were identified in epileptic encephalopathy, using conventional or NGS methods, ${ }^{20}$ with frequencies up to $19.0 \%(16 / 84)$ in Ohtahara syndrome (Table 1). A previous study demonstrated a reduction of $25 \%$ in the $\mathrm{M}$-current amplitude was sufficient to increase neuronal excitability to epileptogenic levels and cause epilepsy. ${ }^{21}$ The majority of variants associated with epileptic encephalopathy exhibited a LOF and dominantnegative effect. $^{22}$ A functional comparison between the R213W and R213Q variants, which were from benign familial neonatal seizures and neonatal epileptic encephalopathy, respectively, demonstrated that $\mathrm{R} 213 \mathrm{Q}$ caused more severe functional impairment, ${ }^{23}$ suggesting that KCNQ2 phenotype severity was correlated with functional deficiency. Several variants associated with epileptic encephalopathy revealed a GOF effect by stabilizing the activated state of the channels, ${ }^{24}$ but their association with epileptic encephalopathy and the underlying mechanism warrant further study.

KCNT1 variants were first reported in autosomal dominant nocturnal frontal lobe epilepsy (ADNFLE). ${ }^{25}$ De novo variants have also been frequently identified in epileptic encephalopathy, mostly in EIMFS $(77.1 \%, 37 / 48)$ (Table 1). All known functional consequences of variants associated with EIMFS and ADNFLE displayed a strong GOF effect by producing large-fold increases in the current amplitude. ${ }^{26-28}$ EIMFSassociated variants caused a significantly larger current than ADNFLE-associated variants. ${ }^{28}$

\section{GABA receptor genes}

$\gamma$-Aminobutyric acid (GABA), the major inhibitory neurotransmitter of the CNS, exerts its action via two types of receptors, i.e., ionotropic $\mathrm{GABA}_{\mathrm{A}}$ and metabotropic $\mathrm{GABA}_{\mathrm{B}}$ receptors. Five genes encoding $\mathrm{GABA}_{\mathrm{A}}$ subunits, including GABRA1, GABRB1, GABRB2, GABRB3, and GABRG2, have been reported in epileptic encephalopathy (Supplementary Tables S4 online).

All known functional consequences of epileptic encephalopathy-associated $\mathrm{GABA}_{\mathrm{A}}$ mutants were LOF (Supplementary Table S8 online), which differed from the diverse functional alterations in sodium or potassium channels. GABRA1, GABRB3, and GABRG2 have been previously reported in mild idiopathic generalized epilepsies, i.e., typically epilepsies characterized by absences. ${ }^{29}$ Their associations with epilepsy were supported by experimental evidence, including studies on genetic knock-out or knock-in models. ${ }^{30-32}$ Using NGS, a number of de novo variants in these genes have been identified in intractable epileptic encephalopathy. Recent studies have demonstrated that GABRG2 variants associated with epileptic encephalopathy had a profound dominant negative effect, in contrast to pure haploinsufficiency in idiopathic generalized epilepsies, explaining the severe epileptic encephalopathy caused by GABRG2 variants. ${ }^{31}$ However, the factors that influence phenotype severity and their roles in epileptic encephalopathy are still elusive for other genes.

\section{Glutamate receptor genes}

$\mathrm{N}$-methyl-D-aspartate receptors are predominant excitatory neurotransmitter receptors. Variants in GRIN1, GRIN2A, GRIN2B, and GRIN2D have been identified in epileptic encephalopathy (Table 1, Supplementary Table S4 online).

To date, 15 de novo heterozygous variants in GRIN1 have been reported in 23 cases, ${ }^{33,34}$ including 7 variants in epileptic encephalopathy (Table 1). Severe intellectual disability associated with profound global developmental delay is a predominant feature of the phenotype. Approximately $70 \%$ of these patients had epilepsy of varying severity. Functional studies revealed a LOF effect of all GRIN1 variants. ${ }^{34}$ Homozygous GRIN1 variants were associated with a more severe phenotype. $^{34}$, suggesting a quantity-dependent feature

More than 60 GRIN2A variants have been reported, mostly associated with the focal epilepsy spectrum. ${ }^{35}$ The majority of 
Table 2 Genotype, functional alteration, and phenotype of variants in non-ion channel genes in epileptic encephalopathies

Funotype (variant EE phenotype (case no.)

no.)

\begin{tabular}{|c|c|c|c|c|}
\hline \multicolumn{5}{|c|}{ Transporter/receptor } \\
\hline ATP1A2 & Missense (2) & UD (2) & UEE (2) & $\mathrm{FHM}^{\mathrm{a}}(\mathrm{AD})$ \\
\hline NTRK2 & Missense (2) & UD (2) & WS (3), uEE (2) & OBHD (de novo) \\
\hline \multirow[t]{2}{*}{$S L C 2 A 1$} & Missense (1) & LOF (1) & UEE (1) & Dystonia $^{a}(A D)$ \\
\hline & Truncating (1) & Destructive (1) & UEE (1) & / \\
\hline \multirow[t]{2}{*}{ SLC6A1 } & Missense (4) & UD (4) & EMAS (4) & / \\
\hline & Truncating (2) & Destructive (2) & EMAS (2) & / \\
\hline STXBP1 & $\begin{array}{l}\text { Truncating (9), splice (6), GR } \\
\text { (4) }\end{array}$ & Destructive (19) & OS (7), WS (2), uEE (11) & $\mathrm{MR}^{\mathrm{a}}$ (de novo) \\
\hline \multicolumn{5}{|c|}{ Signaling molecule } \\
\hline FGF12 & Missense (1) & GOF (1) & uEE (7) & / \\
\hline \multirow[t]{2}{*}{ SPTAN1 } & Missense (1) & UD (1) & UEE (1) & / \\
\hline & Truncating (2) & Destructive (2) & WS (1), UEE (1) & GE (de novo) \\
\hline \multicolumn{5}{|c|}{ Nucleic acid binding protein } \\
\hline ANKRD11 & Truncating (2) & Destructive (2) & WS (1), uEE (1) & $K B G S^{a}$ (AD or de novo) \\
\hline EEF1A2 & Missense (3) & UD (3) & WS (2), LGS (1), uEE (5) & $\mathrm{MR}^{\mathrm{a}}$ (de novo) \\
\hline \multirow[t]{2}{*}{ FOXG1 } & Missense (1) & UD (1) & $\operatorname{LGS}(1)$, WS (1) & $\mathrm{RTT}^{\mathrm{a}}$ (unknown) \\
\hline & Truncating (1) & Destructive (1) & UEE (1) & $\mathrm{RTT}^{\mathrm{a}}$ (de novo) \\
\hline NACC1 & Missense (1) & UD (1) & WS (4) & $\operatorname{NDD}^{a}$ (de novo) \\
\hline \multicolumn{5}{|l|}{ Enzyme } \\
\hline CHD2 & Missense (3) & UD (3) & uEE (3) & I \\
\hline HECW2 & Missense (3) & UD (3) & WS (2), uEE (3) & $\mathrm{NDD}^{\mathrm{a}}$ (de novo) \\
\hline NEDD4L & Missense (2) & UD (2) & WS (2) & PNH (de novo or $A D^{a}$ ) \\
\hline \multicolumn{5}{|c|}{ Enzyme modulator } \\
\hline \multirow[t]{2}{*}{ SYNGAP1 } & Missense (1) & UD (1) & WS (1) & $\mathrm{MR}^{\mathrm{a}}$ (de novo) \\
\hline & Truncating (5) & Destructive (5) & uEE (5) & MR (de novo ${ }^{a}$ or $A D$ ) \\
\hline
\end{tabular}

Genes with pathogenic potential to be confirmed are not listed here. Details in Supplementary Table S 5 online

$A D$ autosomal dominant, DS Dravet syndrome, EE epileptic encephalopathy, EMAS epilepsy with myoclonic-atonic seizures, EMPF encephalopathy due to defective mitochondrial and peroxisomal fission, FHM familial hemiplegic migraine, GE generalized epilepsy, GOF gain of function, GR genomic rearrangement, KBGS KBG syndrome, LGS Lennox-Gastaut syndrome, LOF loss of function, MR mental retardation, NDD neurodevelopmental disorder, NEDIM neurodevelopmental disorder with involuntary movements, OBHD obesity, hyperphagia, and developmental delay, OS Ohtahara syndrome, pLOF partial loss of function, PNH periventricular nodular heterotopia, RTT Rett syndrome, UD functional alteration not determined missense, UEE unspecified epileptic encephalopathy, WS West syndrome

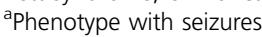

variants were found in families with incomplete penetrance or intrafamilial variability. Previous studies have observed a higher mutation frequency in the more severe phenotype. ${ }^{36}$ Using NGS technologies, three GRIN2A variants were found in epileptic encephalopathy other than Landau-Kleffner syndrome and
ECSWS (Table 1). Most functional studies on GRIN2A mutations demonstrated a GOF effect, ${ }^{37}$ suggesting that GRIN2A variants with GOF may contribute to epileptic encephalopathy, particularly Landau-Kleffner syndrome and ECSWS, whereas the role of variants with other funotypes remains elusive. ${ }^{38}$ 
GRIN2B variants were initially identified in patients with intellectual disability or autism spectrum disorder. ${ }^{39,40}$ Thirteen de novo variants were detected in patients with epileptic encephalopathy from different cohorts (Table 1). The missense variants N615I and V618G from West syndrome dramatically increased $\mathrm{Ca}^{2+}$ influx (GOF), while the $\mathrm{R} 540 \mathrm{H}$ variant from focal epilepsy caused a milder GOF effect, ${ }^{41}$ implying a potential quantitative correlation between the functional alteration and clinical severity.

\section{Other ion channel genes}

HCN1 is one of four genes encoding hyperpolarizationactivated, cyclic nucleotide-gated channels (HCNs) with permeation to $\mathrm{Na}^{+}$and $\mathrm{K}^{+}$( $I_{\mathrm{h}}$ current). A study detected six missense variants in patients with early infantile epileptic encephalopathy characterized as fever-sensitive. ${ }^{42}$ Electrophysiological studies showed either a LOF or GOF effect of the variants (Table 1). ${ }^{42}$ The $H c n 1$ knockout mice displayed increased excitability and sensitivity to convulsants, ${ }^{43}$ supporting the association between HCN1 LOF and epilepsy.

\section{Non-ion channel genes}

A growing number of non-ion channel genes are potentially associated with epileptic encephalopathy. All functionally determined variants in non-ion channel genes caused a LOF, except those in FGF12. Potential epileptic encephalopathy-associated genes included CHD2, DNM1, FGF12, GNAO1, SLC6A1, SPTAN1, and STXBP1 (Table 2, Supplementary Table S5 online).

The CHD2-encoded CHD2 protein is a member of the chromodomain helicase DNA-binding proteins and acts to modulate chromatin structure. ${ }^{44}$ Eleven CHD2 de novo variants have been identified in patients with epileptic encephalopathy such as Lennox-Gastaut syndrome and EMAS. The majority of variants were destructive, suggesting a role of haploinsufficiency in pathogenesis. An in vivo zebrafish model system revealed that $c h d 2$-knockdown larvae exhibited an abnormal movement pattern with epileptiform discharges. ${ }^{45}$

DNM1 encodes dynamin-1, a brain-expressed GTPase, which is involved in activity-dependent synaptic vesicle recycling. ${ }^{46}$ Ten de novo missense variants have been identified in epileptic encephalopathy. Functional studies on A177P, K206N, and G359A revealed the impaired endocytosis of transferrin, in a dominant-negative manner. ${ }^{47}$ A mouse model with heterozygous A408T missense variant developed recurrent limbic and generalized tonic-clonic seizures, while homozygous mice exhibited a more severe phenotype. ${ }^{48}$

FGF12 (also known as FHF1) encodes a small cytosolic protein that interacts with the cytoplasmic tails of voltagegated sodium channels. FGF12 can promote excitability by delaying fast inactivation of the channels. A recurrent de novo FGF12 variant (R52H) was reported recently in seven unrelated patients with epileptic encephalopathy (Table 2). ${ }^{49}$ All affected individuals presented with early-onset intractable seizures, mostly tonic seizures and frequent status epilepticus. Functional studies showed enhanced modulation of channel inactivation gating, equivalent to a GOF effect. ${ }^{49}$ The $f h f 1 b 1$ $\mathrm{R} 56 \mathrm{H}$ (corresponds to $\mathrm{R} 52 \mathrm{H}$ in $\mathrm{h} F G F 12$ ) knock-in zebrafish model displayed recurrent epileptiform discharges in $50 \%$ of larvae, indicating significantly higher epileptic potential than controls. ${ }^{49}$

GNAO1 encodes $\mathrm{Ga}_{\mathrm{o}}$, which is the a-subunit of heterotrimeric guanine nucleotide-binding proteins (G-proteins). De novo heterozygous GNAO1 variants have been identified in Ohtahara syndrome, West syndrome, and other forms of epileptic encephalopathy (Table 2). Transient-expression studies demonstrated that the D174G, T191_F197del, and I279N variants impaired normal protein localization to the plasma membrane. Electrophysiological analysis demonstrated that D174G, T191_F197del, and G203R variants resulted in decreased $\mathrm{Ga}_{0}$-mediated inhibition of calcium currents by norepinephrine. ${ }^{50}$ Furthermore, a Gnao 1 G184S knock-in mouse model displayed seizures and an increased frequency of interictal epileptiform discharges on EEG. $^{51}$

SLC6A1 encodes a voltage-dependent GABA transporter (GAT-1); its expression reflects GABAergic pathways. ${ }^{52}$ Six SLC6A1 variants were identified in seven cases with EMAS from a cohort of 644 patients with epileptic encephalopathy. They accounted for $3.8 \%$ of the probands (6/160) with EMAS (Supplementary Table S5 online). Five of them were de novo variants, among which one was passed on to the next generation with the same phenotype. Another variant was inherited from an unaffected mother of mosaicism, ${ }^{53}$ indicating a potential quantity-dependent feature. It is therefore possible that SLC6A1 variants are specifically associated with EMAS.

SPTAN1 encodes $\alpha$-II spectrin, a filamentous cytoskeletal protein that regulates the stability of axonal structure. To date, eight de novo variants have been identified, including three identified by NGS (Supplementary Table S5 online). A hotspot variant, D2303_L2305 dup, has been identified in three patients with West syndrome. The phenotypes of SPTAN1 variants included epileptic encephalopathy and generalized epilepsy with intellectual disability ${ }^{54}$ Experimental studies showed that variants with deletion or duplication in the last two spectrin repeats could lead to pathological aggregation of $\alpha / \beta$ spectrin heterodimer, and subsequently, the disturbed clustering of ankyrin $G$ and voltage-gated sodium channels at axon initial segment, together with an elevated action potential threshold. ${ }^{55}$ An in-frame deletion variant of SPTAN1 showed less severe consequence of abnormal aggregation and was associated with relatively mild generalized epilepsy with intellectual disability, ${ }^{54}$ suggesting a potential quantitative relationship.

The association between STXBP1 de novo variants and epileptic encephalopathy has been demonstrated in previous studie $^{56,57}$ Recently, more variants have been identified in epileptic encephalopathy, further supporting the role of $S T X B P 1$ variants in epileptic encephalopathy. 


\section{X-linked genes}

$\mathrm{X}$-linked genes are discussed separately because their variants affect male and female patients differently. Because males have one copy of the $\mathrm{X}$ chromosome, while females have two copies, a pathogenic heterozygous variant (hemizygous) in the $\mathrm{X}$ chromosome causes relatively more severe genetic damage in males. Thus, male patients with X-linked gene variants are more severely affected than female patients. Such a quantitydependent feature is an additional consideration in the assessment of pathogenicity of de novo variants.

All X-linked genes in epileptic encephalopathy have previously been reported to be associated with epilepsy or seizures (Supplementary Tables S6, S9 online). Current data from functional studies and the common destructive variants suggest a role of LOF or haploinsufficiency of these genes in the epileptogenesis of epileptic encephalopathy (Supplementary Table S9 online). Differences in phenotype severity between male and female patients were observed in CASK, CDKL5, CLCN4, IQSEC2, SLC35A2, and WDR45 (Supplementary Table S10 online).

CASK, DCX, and PCDH19 are reportedly associated with epileptic encephalopathy previously. ${ }^{58-61}$ Using NGS techniques, novel de novo variants in these genes have been identified in patients with epileptic encephalopathy (Supplementary Table S6, S9 online), suggesting a role of these variants in epileptic encephalopathy. PCDH19 gene shows an unusual X-linked inheritance with highly selective involvement of female patients, except for several male patients with somatic mosaicism. ${ }^{62}$ PCDH19 encodes protocadherin 19, which plays roles in neuronal migration and synaptic plasticity. Most variants arose de novo in the sporadic cases, with a few being transmitted from asymptomatic parents or affected mothers. ${ }^{63}$ Hemizygous males are clinically unaffected. This special phenomenon could be attributed to a mechanism known as "cellular interference," 61 i.e., a heterogeneous population of mutant and wildtype cells are required for disease to occur; while a homogeneous cell population (either mutant or wildtype) does not lead to disease.

CDKL5/STK9 encodes a serine/threonine kinase protein with a high expression level in mature neurons in the cerebral cortex and hippocampus. CDKL5 variants have been reported in diverse phenotypes, including Rett syndrome, epileptic encephalopathy, and autism. ${ }^{58,64}$ In previous studies, the majority of CDKL5 variants have been identified in girls, with only a few being reported in boys. CDKL5 variants were found in an estimated $8-16 \%$ of girls with early onset seizures, and more specifically in $28 \%$ of girls with early onset seizures combined with West syndrome. ${ }^{64}$ However, affected boys showed a more severe clinical manifestation. ${ }^{64}$ Using NGS, an additional 51 de novo variants were identified in severe epileptic encephalopathy cases, which included quite a few boys (Supplementary Table S6 online).

CLCN4 encodes the voltage-dependent $2 \mathrm{Cl}^{-} / \mathrm{H}^{+}$-exchanger ClC-4. CLCN4 variants have been previously identified in families with X-linked intellectual disability and seizures, ${ }^{65,66}$ and affected male patients had a more severe phenotype than female patients. ${ }^{66}$ The identification of two de novo variants in boys with severe epileptic encephalopathy further supported the pathogenic role of CLCN4 (Supplementary Table S6 online).

IQSEC2 encodes a guanine nucleotide exchange factor for the ADP ribosylation factors family of small GTP-binding proteins. Its variants have been previously implicated in Xlinked intellectual disability. Moreover, epilepsy is a common finding in the patients. ${ }^{67}$ Boys usually have a more severe phenotype than girls. ${ }^{67}$ Ten de novo IQSEC2 variants were recently identified in patients with epileptic encephalopathy, including four variants in male patients (Supplementary Tables S6 online).

SLC35A2 encodes a Golgi-localized UDP-galactose transporter, which imports UDP-galactose from the cytoplasm to the lumen of the Golgi apparatus. SLC35A2 was reported previously in four patients with congenital disorder of glycosylation, among whom three showed seizures and hypsarrhythmia on EEGs. ${ }^{68,69}$ Recently, nine de novo SLC35A2 variants were identified in patients with epileptic encephalopathy (Supplementary Table S6 online), accompanied by characteristic hypsarrhythmia on EEGs. ${ }^{69,70}$

WDR45, which encodes a WD40-repeat protein that plays an important role in the autophagy pathway, is associated with a subtype of neurodegeneration with brain iron accumulation. $^{71}$ Six novo variants have been reported in patients with epileptic encephalopathy (Supplementary Table S6 online), among whom boys were more severely affected. $^{72,73}$

\section{EVIDENCE IMPLICATING THE PATHOGENIC POTENTIAL OF GENES}

In analyzing the associations between epileptic encephalopathy and the genes, evidence from several aspects indicated a general association between epilepsy and the genes, which were considered to support a possibly pathogenic role of the genes in epileptic encephalopathy. These include the following: (1) significantly high frequency or hotspot of variants in the patient group, (2) other epilepsy-related phenotype with defined inheritance pattern, (3) variants in the gene result in functional impairment that is related to epilepsy. Genes with at least one of these characteristics (Table 3) were considered possibly pathogenic.

Generally, a significantly higher frequency of variant helps determine the association between a gene and a genetic disease. ${ }^{3}$ De novo variants were identified in rare cases of each independent epileptic encephalopathy cohort, which varied considerably in size (Supplementary Tables S2-7 online). Therefore, except the de novo SCN1A variants in Dravet syndrome cases, ${ }^{6}$ it was difficult to determine the frequency of de novo variants in most epileptic encephalopathies. However, hotspot variants were found in several genes (Table 3 ).

Familial epilepsies with dominant inheritance have been reported previously in several genes (Table 3), supporting their possible roles in epileptic encephalopathy. Theoretically, although a heterozygous de novo variant may compose a 
homozygous (or compound heterozygous) variant with an inherited variant and present a recessive inheritance pattern, ${ }^{74}$ this has not been observed in epileptic encephalopathy.

Functional studies on variants have been performed for most ion-channel genes and several non-ion channel genes. The functional impairments explained epileptogenesis in the majority of the epileptic encephalopathy-related genes, such as LOF of GABAergic receptors. However, the funotype of variants in several genes could not explain the occurrence of epilepsy, such as the LOF of the SCN8A gene. ${ }^{14}$

Evidence with several characteristics was found to be specific in supporting the pathogenic role of genes in epileptic encephalopathy. This included the following: (1) variants of the gene are associated with characteristic phenotype; (2) distinct correlation between genotype/funotype and phenotype; (3) correlation between genetic impairment and phenotype severity, explaining the severe phenotype of epileptic encephalopathy; and (4) variant-specific model with corresponding phenotype or defined mechanisms of epileptogenesis. Supplementary Table S10 online summarizes the evidence for such genes with de novo variants, which were recurrently identified in unrelated cases.

First, two genes are associated with characteristic epileptic encephalopathy phenotypes. SLC6A1 mutations were identified in EMAS cases alone, but not in cases with other forms of epileptic encephalopathy. ${ }^{53}$ SLC $35 \mathrm{~A} 2$ variants were associated with West syndrome with hypsarrhythmia on EEGs. ${ }^{69,70}$

Second, variations of several ion channel genes elicited diverse functional alterations, including those in GRIN2A, GRIN2B, HCN1, KCNA2, KCNQ2, SCN1A, SCN2A, and SCN8A. Distinct funotype-phenotype correlation could be found in $S C N 1 A$ and KCNA2. All epileptic encephalopathy-related $S C N 1 A$ variants were featured by LOF, whereas GOF variants were associated with mild generalized epilepsy with febrile seizures plus. ${ }^{6}$ Similarly, KCNA2 LOF variants were associated with partial epilepsy that responded well to antiepileptic treatment, in contrast to GOF variants that tended to cause generalized epilepsy with less favorable outcomes. ${ }^{16}$

Third, a correlation between genetic impairment and phenotype severity was found in several genes, explaining the severity of epileptic encephalopathy (Supplementary Table S10 online).

Fourth, variant-specific knock-in models have produced epileptic encephalopathy for several genes, or epileptogenesis mechanism has been defined, providing direct evidence of their causative role in epileptic encephalopathy (Supplementary Table S10 online).

Based on the analysis of evidence supporting pathogenicity, we summarized the pathogenic potential of epileptic encephalopathy-associated genes and their pathogenic genotype and funotype (Table 4). A pathogenic or possible pathogenic role with defined funotype could be validated in 51 genes, whereas the pathogenic role of 23 genes warrants
Table 3 Summary of evidence implicating possibly pathogenic genes in epileptic encephalopathies

\begin{tabular}{|c|c|c|c|}
\hline Gene & $\begin{array}{l}\text { Hotspot variant } \\
\text { (no.) }\end{array}$ & $\begin{array}{l}\text { Other dominantly } \\
\text { inherited epilepsy } \\
\text { phenotype }\end{array}$ & $\begin{array}{l}\text { Funotype } \\
\text { related to } \\
\text { epilepsy }\end{array}$ \\
\hline \multicolumn{4}{|c|}{ Ion channel } \\
\hline \multirow[t]{2}{*}{ SCN2A } & A263 (4), R853 (4), & BFNS & GOF \\
\hline & $\begin{array}{l}\text { E999 (3), L1342 (3), } \\
\text { R1882 (5) }\end{array}$ & BFNS & LOF? \\
\hline \multirow[t]{2}{*}{ SCN8A } & R850 (3), R1617 (4), & BFIS (UD) & GOF \\
\hline & $\begin{array}{l}\text { A1650 (3), R1872 } \\
(13)\end{array}$ & & LOF? \\
\hline KCNA1 & / & EA/myokymia ${ }^{\mathrm{a}}(\mathrm{UD})$ & LOF? \\
\hline \multirow[t]{2}{*}{ KCNA2 } & R297 (7), T374 (3), & $\mathrm{HSP} \mathrm{P}^{\mathrm{a}}$ & LOF \\
\hline & P405 (4) & l & GOF? \\
\hline CACNA1A & A713 (3) & EA/FHM ${ }^{a}$ & I \\
\hline GABRA1 & M263 (3) & IGE，JME & LOF \\
\hline GABRB1 & I & I & LOF \\
\hline GABRB2 & Y244 (4) & / & LOF \\
\hline GABRB3 & I & CAE & LOF \\
\hline \multirow[t]{2}{*}{ GRIN2A } & l & $\mathrm{FE}$ & GOF \\
\hline & & $\mathrm{FE}$ & LOF? \\
\hline GRIN2D & l & / & GOF \\
\hline \multirow[t]{2}{*}{ HCN1 } & I & I & LOF \\
\hline & l & / & GOF? \\
\hline \multicolumn{4}{|c|}{ Non-ion channel } \\
\hline ANKRD11 & l & $\mathrm{KBGS}^{\mathrm{a}}$ & I \\
\hline ATP1A2 & I & $\mathrm{FHM}^{\mathrm{a}}$ & I \\
\hline DNM1L & l & l & LOF \\
\hline DYNC1H1 & l & $\mathrm{MR}^{\mathrm{a}}$ & l \\
\hline EEF1A2 & G70 (4), E122 (3) & / & I \\
\hline FOXG1 & l & $\mathrm{RTT}^{\mathrm{a}}$ & I \\
\hline HECW2 & R1330 (3) & I & I \\
\hline NACC1 & R298 (4) & l & I \\
\hline NEDD4L & / & $\mathrm{PNH}^{\mathrm{a}}(\mathrm{UD})$ & / \\
\hline NTRK2 & Y434 (4) & 1 & I \\
\hline$S L C 2 A 1$ & I & Dystonia $^{a}$ & LOF \\
\hline SYNGAP1 & 1 & I & LOF \\
\hline YWHAG & R132 (3) & I & I \\
\hline \multicolumn{4}{|l|}{ X-linked } \\
\hline$A L G 13$ & N107 (6) & I & I \\
\hline MECP2 & I & $\mathrm{RTT} \mathrm{T}^{\mathrm{a}}$ & LOF \\
\hline PIGA & I & I & LOF \\
\hline SLC9A6 & I & $\mathrm{MR}^{\mathrm{a}}$ & I \\
\hline
\end{tabular}

Hotspot variants listed here are those that have been observed in no less than three unrelated cases

BFIS benign familial infantile seizures, BFNS benign familial infantile seizures, CAE childhood absence epilepsy, EA episodic ataxia, FE focal epilepsy, FHM familial hemiplegic migraine, GOF gain of function, HSP hereditary spastic paraplegia, IGE idiopathic generalized epilepsies, JME juvenile myoclonic epilepsy, KBGS KBG syndrome, LOF loss of function, MR mental retardation, $P N H$ periventricular nodular heterotopia, RTT Rett syndrome, UD functional alteration not determined missense

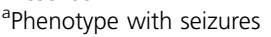


Table 4 Summary of pathogenic potential, functional alteration, and genotype of genes in epileptic encephalopathies Pathogenic Possibly pathogenic To be confirmed

\begin{tabular}{|c|c|c|c|}
\hline GOF & Ion channel & & \\
\hline \multirow[t]{2}{*}{ (missense) } & $\begin{array}{l}\text { KCNA2, KCNT1; GRIN2A, } \\
\text { GRIN2B }\end{array}$ & SCN2A, SCN8A; GRIN2D & KCNQ2; HCN1 \\
\hline & FGF12 & & \\
\hline LOF or & Ion channel & & \\
\hline \multirow[t]{4}{*}{ destructive } & $\begin{array}{l}\text { SCN1A; KCNA2 }{ }^{\mathrm{a}}, K^{\prime} C N B 1, \\
\text { KCNQ2; GABRG2 }^{\mathrm{a}} \text { GRIN1 }\end{array}$ & $\begin{array}{l}\text { SCN2A; GABRA1, GABRB1 }{ }^{\mathrm{a}}, \mathrm{GABRB2^{ \textrm {a } }}, \\
\text { GABRB3; GRIN2A }{ }^{\mathrm{a}} ; \mathrm{HCN1}^{\mathrm{a}}\end{array}$ & SCN8A; GRIN2B \\
\hline & $\begin{array}{l}\text { CHD2, DNM1 }{ }^{\mathrm{a}}, \text { GNAO1, } \\
\text { SLC6A1, SPTAN1, STXBP1 }\end{array}$ & ANKRD11, DNM1L ${ }^{\mathrm{a}}, \mathrm{SLC2A1}$, SYNGAP1 & DYRK1A, HNRNPU, KMT2A, NUS1, TRIM8, ZEB2 \\
\hline & X-linked & & \\
\hline & $\begin{array}{l}\text { CASK, CDKL5, CLCN4 } \\
\text { DCX, IQSEC2, PCDH19 } \\
\text { SLC35A2, WDR45 }\end{array}$ & MECP2, SLC9A6 & SMC1A \\
\hline
\end{tabular}

Underline, gene with diverse functional alterations

GOF gain of function, LOF loss of function, UD functional alteration not determined missense

${ }^{a}$ Genes with variants of only missense that revealed a functional alteration of LOF

bspecial mechanism other than LOF and GOF

Table 5 Guideline for evaluation of pathogenic potential of genes with de novo variants in epileptic encephalopathies Genes with de novo variants recurrently identified in unrelated cases are subject to further evaluation of pathogenic potential.

Possibly pathogenic with at least one of the following evidence:

1. Significantly high frequency or hotspot of variants in the patient group.

2. Other epilepsy-related phenotype with defined inheritance pattern.

3. Variants in the gene result in functional impairment that is related to epilepsy.

Pathogenic with at least one of the following specific evidence:

1. Variants of the gene are associated with characteristic phenotype.

2. Distinct correlation between genotype/funotype and phenotype.

3. Correlation between genetic impairment and phenotype severity, and it explains the severe phenotype.

4. Variant-specific model with corresponding phenotype, or defined mechanisms of epileptogenesis.

further validation. Variants of diverse functional alterations were found in several genes; and in four of these genes, the pathogenicity of one of the funotypes could not be determined. Table 5 summarizes the evidence implicating the pathogenic potential of genes associated with epileptic encephalopathies.

\section{SUMMARY}

The evaluation of de novo heterozygous variants identified in patients with epileptic encephalopathy is a major challenge. Current guidelines have provided useful, but relatively general, tools for evaluating the causality of these variants. $^{3,4,75}$ However, epileptic encephalopathies are specific age-related conditions with heterogeneous clinical phenotypes, multiple etiologies, and complex mechanisms of epileptogenesis. Therefore, the evaluation of the pathogenic potential of epileptic encephalopathy-related genes, i.e., the pathogenicity at gene level, is critical. The present data showed that previously reported epileptic encephalopathy- related genes differ widely in their association with epileptic encephalopathy. Based on currently available data, 24 genes were estimated to be pathogenic and 27 genes were estimated to be possibly pathogenic in epileptic encephalopathies. Furthermore, epileptic encephalopathy-related genes had specific pathogenic funotypes, such as GOF KCNT1 variants and LOF GABRG2 variants. Even genes with a similar function and structure or ones that involved in the same functional pathway, such as sodium channel genes SCN1A, $S C N 2 A$, and SCN8A, however, differ in their pathogenic potential in epileptic encephalopathies, including their pathogenic genotypes and funotypes. A total of 23 genes require further study to determine their pathogenic role or pathogenic funotype in epileptic encephalopathy. Thus, the pathogenicity of variants in these genes should be considered conservatively. The data suggest that evaluating the pathogenic potential of genes is required before considering the pathogenicity of variants in a clinical or genetic consulting 
practice. The summary of epileptic encephalopathy-associated genes and their pathogenic genotype and funotype (Table 4) may be used to help evaluate the pathogenicity of variants identified in an individual with epileptic encephalopathy.

Based on evidence in supporting pathogenicity, we outlined a guideline for evaluating the pathogenic potential of genes associated with epileptic encephalopathy (Table 5). From a clinical genetics point of view, characteristic phenotype, distinct genotype/funotype-phenotype correlation, and correlation between genetic impairment and phenotype severity appeared to be specific considerations when evaluating the pathogenic potential of genes related to epileptic encephalopathy. The summarized data is expected to be helpful in outlining guidelines for evaluating the pathogenic potential of genes associated with other genetic diseases.

\section{ELECTRONIC SUPPLEMENTARY MATERIAL}

The online version of this article (https://doi.org/10.1038/s41436018-0011-y) contains supplementary material, which is available to authorized users.

\section{ACKNOWLEDGEMENTS}

This work was supported by omics-based precision medicine of epilepsy being entrusted by Key Research Project of the Ministry of Science and Technology of China (grant no. 2016YFC0904400), the National Natural Science Foundation of China (grant nos. 81501125, 81571273, and 81571274), the Natural Science Foundation of Guangdong Province (grant no. 2014A030310094), the Cultivation and Innovation Fund of Jinan University (grant no. 2161533), and Science and Technology Project of Guangzhou (grant nos. 201508020011, 201604020161, and 201607010002). The funders had no role in study design, data collection, data analysis, and decision to prepare or publish the manuscript.

\section{DISCLOSURE}

The authors declare no conflicts of interest.

\section{REFERENCES}

1. Eltze CM, Chong WK, Cox T, et al. A population-based study of newly diagnosed epilepsy in infants. Epilepsia. 2013;54:437-45.

2. McTague A, Howell KB, Cross JH, Kurian MA, Scheffer IE. The genetic landscape of the epileptic encephalopathies of infancy and childhood. Lancet Neurol. 2016;15:304-16.

3. MacArthur DG, Manolio TA, Dimmock DP, et al. Guidelines for investigating causality of sequence variants in human disease. Nature. 2014;508:469-76.

4. Richards S, Aziz N, Bale S, et al. Standards and guidelines for the interpretation of sequence variants: a joint consensus recommendation of the American College of Medical Genetics and Genomics and the Association for Molecular Pathology. Genet Med. 2015;17:405-24.

5. Gussow AB, Petrovski S, Wang Q, Allen AS, Goldstein DB. The intolerance to functional genetic variation of protein domains predicts the localization of pathogenic variants within genes. Genome Biol. 2016:17:9.

6. Meng $\mathrm{H}, \mathrm{Xu} \mathrm{HQ}, \mathrm{Yu}$ L, et al. The SCN1A variant database: updating information and analysis of the relationships among genotype, functional alteration, and phenotype. Hum Mutat. 2015;36:573-80.
7. Wei F, Yan LM, Su T, et al. Ion channel genes and epilepsy: functional alteration, pathogenic potential, and mechanism of epilepsy. Neurosci Bull. 2017;33:455-77.

8. Wang J, Lin Z, Liu L, et al. Epilepsy-associated genes. Seizure. 2017;44:11-20.

9. Veltman JA, Brunner HG. De novo variants in human genetic disease. Nat Rev Genet. 2012;13:565-75.

10. Escayg A, MacDonald BT, Meisler MH, et al. Variants of SCN1A, encoding a neuronal sodium channel, in two families with GEFS + 2. Nat Genet. 2000;24:343-5

11. Berkovic SF, Heron SE, Giordano L, et al. Benign familial neonatalinfantile seizures: characterization of a new sodium channelopathy. Ann Neurol. 2004;55:550-7.

12. Shi $X$, Yasumoto $S$, Kurahashi $H$, et al. Clinical spectrum of SCN2A variants. Brain Dev. 2012;34:541-5.

13. Planells-Cases R, Caprini M, Zhang J, et al. Neuronal death and perinatal lethality in voltage-gated sodium channel alpha(II)-deficient mice. Brain Dev. Biophys J. 2000;78:2878-91.

14. Martin MS, Tang B, Papale LA, Yu FH, Catterall WA, Escayg A. The voltage-gated sodium channel Scn8a is a genetic modifier of severe myoclonic epilepsy of infancy. Hum Mol Genet. 2007;16:2892-9.

15. Kohling R, Wolfart J. Potassium channels in epilepsy. Cold Spring Harb Perspect Med. 2016;6:a022871.

16. Masnada S, Hedrich UBS, Gardella E, et al. Clinical spectrum and genotype-phenotype associations of KCNA2-related encephalopathies. Brain. 2017;140:2337-54.

17. Torkamani $A$, Bersell $K$, Jorge $B S$, et al. De novo KCNB1 variants in epileptic encephalopathy. Ann Neurol. 2014;76:529-40.

18. Saitsu H, Akita T, Tohyama J, et al. De novo KCNB1 variants in infantile epilepsy inhibit repetitive neuronal firing. Sci Rep. 2015;5:15199.

19. Villa C, Combi R. Potassium channels and human epileptic phenotypes: an updated overview. Front Cell Neurosci. 2016;10:81.

20. Weckhuysen S, Mandelstam S, Suls A, et al. KCNQ2 encephalopathy: emerging phenotype of a neonatal epileptic encephalopathy. Ann Neurol. 2012;71:15-25.

21. Jentsch TJ. Neuronal KCNQ potassium channels: physiology and role in disease. Nat Rev Neurosci. 2000;1:21-30.

22. Orhan $G$, Bock $M$, Schepers $D$, et al. Dominant-negative effects of KCNQ2 variants are associated with epileptic encephalopathy. Ann Neurol. 2014;75:382-94.

23. Miceli F, Soldovieri MV, Ambrosino $P$, et al. Genotype-phenotype correlations in neonatal epilepsies caused by variants in the voltage sensor of K(v)7.2 potassium channel subunits. Proc Natl Acad Sci U S A. 2013;110:4386-91.

24. Miceli F, Soldovieri MV, Ambrosino P, et al. Early-onset epileptic encephalopathy caused by gain-of-function variants in the voltage sensor of Kv7.2 and Kv7.3 potassium channel subunits. I Neurosci. 2015:35:3782-93.

25. Heron SE, Smith KR, Bahlo M, et al. Missense variants in the sodiumgated potassium channel gene KCNT1 cause severe autosomal dominant nocturnal frontal lobe epilepsy. Nat Genet. 2012;44:1188-90.

26. Kim GE, Kronengold J, Barcia G, et al. Human slack potassium channel variants increase positive cooperativity between individual channels. Cell Rep. 2014;9:1661-72.

27. Barcia G, Fleming MR, Deligniere A, et al. De novo gain-of-function KCNT1 channel variants cause malignant migrating partial seizures of infancy. Nat Genet. 2012;44:1255-9.

28. Milligan CJ, Li M, Gazina EV, et al. KCNT1 gain of function in 2 epilepsy phenotypes is reversed by quinidine. Ann Neurol. 2014;75:581-90.

29. Hirose $S$. Mutant $G A B A(A)$ receptor subunits in genetic (idiopathic) epilepsy. Prog Brain Res. 2014;213:55-85.

30. Arain FM, Boyd KL, Gallagher MJ. Decreased viability and absence-like epilepsy in mice lacking or deficient in the GABAA receptor alpha1 subunit. Epilepsia. 2012;53:e161-165.

31. Kang JQ, Shen W, Zhou C, Xu D, Macdonald RL. The human epilepsy variant GABRG2(Q390X) causes chronic subunit accumulation and neurodegeneration. Nat Neurosci. 2015;18:988-96.

32. Reid CA, Kim T, Phillips AM, et al. Multiple molecular mechanisms for a single GABAA variant in epilepsy. Neurology. 2013;80:1003-8.

33. Hamdan FF, Gauthier J, Araki Y, et al. Excess of de novo deleterious variants in genes associated with glutamatergic systems in nonsyndromic intellectual disability. Am J Human Genet. 2011;88:306-16.

34. Lemke JR, Geider K, Helbig KL, et al. Delineating the GRIN1 phenotypic spectrum: a distinct genetic NMDA receptor encephalopathy. Neurology. 2016;86:2171-8. 
35. Burnashev N, Szepetowski P. NMDA receptor subunit variants in neurodevelopmental disorders. Curr Opin Pharmacol. 2015;20:73-82.

36. Lemke JR, Lal D, Reinthaler EM, et al. Variants in GRIN2A cause idiopathic focal epilepsy with rolandic spikes. Nat Genet. 2013;45:1067-72.

37. Yuan $\mathrm{H}$, Hansen $\mathrm{KB}$, Zhang J, et al. Functional analysis of a de novo GRIN2A missense variant associated with early-onset epileptic encephalopathy. Nat Commun. 2014;5:3251.

38. Addis L, Virdee JK, Vidler LR, Collier DA, Pal DK, Ursu D. Epilepsy-associated GRIN2A variants reduce NMDA receptor trafficking and agonist potency-molecular profiling and functional rescue. Sci Rep. 2017;7:66.

39. Endele S, Rosenberger G, Geider K, et al. Variants in GRIN2A and GRIN2B encoding regulatory subunits of NMDA receptors cause variable neurodevelopmental phenotypes. Nat Genet. 2010;42:1021-6.

40. O'Roak BJ, Vives L, Fu W, et al. Multiplex targeted sequencing identifies recurrently mutated genes in autism spectrum disorders. Science. 2012;338:1619-22.

41. Lemke JR, Hendrickx R, Geider K, et al. GRIN2B variants in West syndrome and intellectual disability with focal epilepsy. Ann Neurol. 2014;75:147-54.

42. Nava C, Dalle $C$, Rastetter A, et al. De novo variants in HCN1 cause early infantile epileptic encephalopathy. Nat Genet. 2014;46:640-5.

43. Huang Z, Walker MC, Shah MM. Loss of dendritic HCN1 subunits enhances cortical excitability and epileptogenesis. J Neurosci. 2009;29:10979-88.

44. Hall JA, Georgel PT. CHD proteins: a diverse family with strong ties. Biochem Cell Biol. 2007;85:463-76.

45. Suls $A$, Jaehn JA, Kecskes $A$, et al. De novo loss-of-function variants in CHD2 cause a fever-sensitive myoclonic epileptic encephalopathy sharing features with Dravet syndrome. Am J Hum Genet. 2013:93:967-75.

46. Ferguson SM, Brasnjo G, Hayashi M, et al. A selective activity-dependent requirement for dynamin 1 in synaptic vesicle endocytosis. Science. 2007:316:570-4.

47. Dhindsa RS, Bradrick SS, Yao X, et al. Epileptic encephalopathy-causing variants in DNM1 impair synaptic vesicle endocytosis. Neurol Genet. 2015;1:e4

48. Boumil RM, Letts VA, Roberts $M C$, et al. A missense variant in a highly conserved alternate exon of dynamin-1 causes epilepsy in fitful mice. PLoS Genet. 2010;6:e1001046.

49. Siekierska A, Isrie M, Liu Y, et al. Gain-of-function FHF1 variant causes early-onset epileptic encephalopathy with cerebellar atrophy. Neurology. 2016;86:2162-70

50. Nakamura $\mathrm{K}$, Kodera $\mathrm{H}$, Akita $\mathrm{T}$, et al. De novo variants in GNAO1, encoding a Galphao subunit of heterotrimeric $\mathrm{G}$ proteins, cause epileptic encephalopathy. Am J Hum Genet. 2013;93:496-505.

51. Kehrl JM, Sahaya K, Dalton HM, et al. Gain-of-function variant in Gnao1: a murine model of epileptiform encephalopathy (EIEE17)? Mamm Genome. 2014;25:202-10.

52. Scimemi A. Structure, function, and plasticity of GABA transporters. Front Cell Neurosci. 2014:8:161.

53. Carvill GL, McMahon JM, Schneider A, et al. Variants in the GABA transporter SLC6A1 cause epilepsy with myoclonic-atonic seizures. Am J Hum Genet. 2015;96:808-15.

54. Tohyama J, Nakashima M, Nabatame S, et al. SPTAN1 encephalopathy: distinct phenotypes and genotypes. J Hum Genet. 2015;60:167-73.

55. Saitsu H, Tohyama J, Kumada T, et al. Dominant-negative variants in alpha-Il spectrin cause West syndrome with severe cerebral hypomyelination, spastic quadriplegia, and developmental delay. Am J Hum Genet. 2010;86:881-91.

56. Mignot C, Moutard ML, Trouillard O, et al. STXBP1-related encephalopathy presenting as infantile spasms and generalized tremor in three patients. Epilepsia. 2011;52:1820-7.

57. Saitsu H, Kato M, Okada I, et al. STXBP1 variants in early infantile epileptic encephalopathy with suppression-burst pattern. Epilepsia. 2010;51:2397-405.

58. Trump N, McTague A, Brittain $\mathrm{H}$, et al. Improving diagnosis and broadening the phenotypes in early-onset seizure and severe developmental delay disorders through gene panel analysis. J Med Genet. 2016;53:310-7.
59. Nakajiri T, Kobayashi K, Okamoto N, et al. Late-onset epileptic spasms in a female patient with a CASK variant. Brain Dev. 2015;37:919-23.

60. Lawrence KM, Mei D, Newton MR, Leventer RJ, Guerrini R, Berkovic SF. Familial Lennox-Gastaut syndrome in male siblings with a novel DCX variant and anterior pachygyria. Epilepsia. 2010;51:1902-5.

61. Depienne C, Bouteiller D, Keren B, et al. Sporadic infantile epileptic encephalopathy caused by variants in PCDH19 resembles Dravet syndrome but mainly affects females. PLoS Genet. 2009:5:e1000381.

62. de Lange IM, Rump P, Neuteboom RF, et al. Male patients affected by mosaic PCDH19 variants: five new cases. Neurogenetics. 2017; 18:147-53.

63. Depienne $C$, Trouillard $\mathrm{O}$, Bouteiller $\mathrm{D}$, et al. Variants and deletions in PCDH19 account for various familial or isolated epilepsies in females. Hum Mutat. 2011;32:E1959-1975.

64. Guerrini R, Parrini E. Epilepsy in Rett syndrome, and CDKL5- and FOXG1gene-related encephalopathies. Epilepsia. 2012;53:2067-78.

65. Hu H, Haas SA, Chelly J, et al. X-exome sequencing of 405 unresolved families identifies seven novel intellectual disability genes. Mol Psychiatry. 2016:21:133-48.

66. Palmer EE, Stuhlmann T, Weinert S, et al. De novo and inherited variants in the X-linked gene CLCN4 are associated with syndromic intellectual disability and behavior and seizure disorders in males and females. Mol Psychiatry. 2016;23:222-30.

67. Zerem A, Haginoya $K$, Lev $D$, et al. The molecular and phenotypic spectrum of IQSEC2-related epilepsy. Epilepsia. 2016;57:1858-69.

68. Dorre $K$, Olczak $M$, Wada $Y$, et al. A new case of UDP-galactose transporter deficiency (SLC35A2-CDG): molecular basis, clinical phenotype, and therapeutic approach. J Inherit Metab Dis. 2015;38:931-40.

69. Ng BG, Buckingham KJ, Raymond K, et al. Mosaicism of the UDPgalactose transporter SLC35A2 causes a congenital disorder of glycosylation. Am J Hum Genet. 2013;92:632-6.

70. Kodera $\mathrm{H}$, Nakamura $\mathrm{K}$, Osaka $\mathrm{H}$, et al. De novo variants in SLC35A2 encoding a UDP-galactose transporter cause early-onset epileptic encephalopathy. Hum Mutat. 2013;34:1708-14.

71. Haack TB, Hogarth $P$, Kruer $M C$, et al. Exome sequencing reveals de novo WDR45 variants causing a phenotypically distinct, $X$-linked dominant form of NBIA. Am J Hum Genet. 2012;91:1144-9.

72. Nakashima M, Takano K, Tsuyusaki $Y$, et al. WDR45 variants in three male patients with West syndrome. J Hum Genet. 2016;61:653-61.

73. Xixis $\mathrm{KI}$, Mikati MA. Epileptic spasms: a previously unreported manifestation of WDR45 gene variant. Epileptic Disord. 2015;17:467-72.

74. Saito T, Ishii A, Sugai K, Sasaki M, Hirose S. A de novo missense variant in SLC12A5 found in a compound heterozygote patient with epilepsy of infancy with migrating focal seizures. Clin Genet. 2017;92:654-8.

75. Rehm HL, Berg JS, Brooks LD, et al. ClinGen-the clinical genome resource. N Engl J Med. 2015;372:2235-42.

\section{(i)(2)}

Open Access This article is licensed under a Creative Commons Attribution-NonCommercial-ShareAlike 4.0 International License, which permits any non-commercial use, sharing, adaptation, distribution and reproduction in any medium or format, as long as you give appropriate credit to the original author(s) and the source, provide a link to the Creative Commons license, and indicate if changes were made. If you remix, transform, or build upon this article or a part thereof, you must distribute your contributions under the same license as the original. The images or other third party material in this article are included in the article's Creative Commons license, unless indicated otherwise in a credit line to the material. If material is not included in the article's Creative Commons license and your intended use is not permitted by statutory regulation or exceeds the permitted use, you will need to obtain permission directly from the copyright holder. To view a copy of this license, visit http://creativecommons.org/licenses/by-nc-sa/4.0/.

(c) The Author(s) 2018 